\title{
Residual Stress Measurement of the Engineering Plastics by the Hole-Drilling Strain-Gage Method
}

\author{
Ami Kohri ${ }^{1, a,{ }^{*}}$, Takao Mikami ${ }^{2, b}$ and Yuhei Suzuki ${ }^{1, c}$ \\ ${ }^{1} \mathrm{IHI}$ Inspection \& Instrumentation Co., Ltd., 6-17, Fukuura, 2-chome, Kanazawa-ku, Yokohama- \\ city, Kanagawa 236-0004, Japan \\ 2 IHI Inspection \& Instrumentation Co., Ltd., 25-3, Minami-Ohi 6-chome, Shinagawa-ku, \\ Tokyo 140-0013, Japan \\ aa_kohri@iic.ihi.co.jp, bt_mikami@iic.ihi.co.jp, y﹎suzuki@iic.ihi.co.jp
}

Keywords: ASTM E837, Hole-Drilling, Plastics, Orthotropic Material, Injection Molding

\begin{abstract}
The hole-drilling method is regulated as the ASTM E837 standard and has been widely used for the residual stress measurement of metallic materials. We applied this method to orthotropic plastic specimens. Known stress distributions with depth were given by the four points bending tests and the calculated stresses from the measured relieved strains were compared with the given values analyzed by the beam theory. Consequently, the calculated uniform stresses based on both orthotropic and isotropic approaches were equivalent to the beam theory analyses. In addition, the calculated non-uniform stresses with depth in accordance with ASTM E837 standard agreed well with the beam theory values. It was confirmed that the residual stress measurement by the hole-drilling method is effective for the orthotropic plastic materials.
\end{abstract}

\section{Introduction}

In recent years, high performance plastic materials with superior mechanical properties and high thermal stability have been developed. They are called 'engineering plastics' and have been applied to industrial products. Particularly, in the field of automotive and aerospace industries glass-fiber reinforced plastics have been actively applied. Most of them have anisotropic elastic properties due to their molding processes. Residual stresses are present in these plastics without exception and are to be measured from the point of view of the quality control.

There are many methods for measuring residual stresses. The hole-drilling method is considered to be the most suitable one for plastic materials. This method is standardized as the ASTM Standard E837 [1] and has been widely used for the residual stress measurement of elastic materials. However this method is limited to isotropic materials. Schajer et al. [2] and Pagliaro et al. [3] have generalized the computational procedures for the hole-drilling method to extend the use of the method to orthotropic materials.

In this study, known bending stresses were given by four point bending tests on the rectangular plate specimens made of glass-fiber reinforced plastics and the measured stresses by the hole-drilling method were compared with the analyzed values based on the beam theory.

\section{Experiment}

Specimen. The material of the specimen is Polyamide 6 (PA6) added with $30 \%$ of glass fiber. After manufacturing a plate $(150 \mathrm{~mm} \times 150 \mathrm{~mm} \times 4 \mathrm{~mm})$ by injection molding, it was cut into rectangular specimens $(110 \mathrm{~mm} \times 22 \mathrm{~mm} \times 4 \mathrm{~mm})$ with longitudinal axis aligned with material direction (MD).

Four point bending test. The residual stresses were simulated by applying a bending load. For this purpose, the rectangular specimen was loaded by using a four point testing apparatus with a 
span of $80 \mathrm{~mm}$ and a loading point distance of $30 \mathrm{~mm}$ as shown in Fig.1. We conducted the tests for two specimens and maximum bending stresses of $15 \mathrm{MPa}$ and $20 \mathrm{MPa}$ were given on the surfaces of them respectively. TML's FRS2-23 rosette strain gages with gages 1, 2, 3 oriented at $0^{\circ} / 135^{\circ} / 270^{\circ}$ respect to $\mathrm{x}$ axis (see Fig. 2) were attached to tensile side surface of each bending specimen. The applied bending stress was monitored by two strain gages aligned with longitudinal direction of each specimen during the test (see Fig. 2).

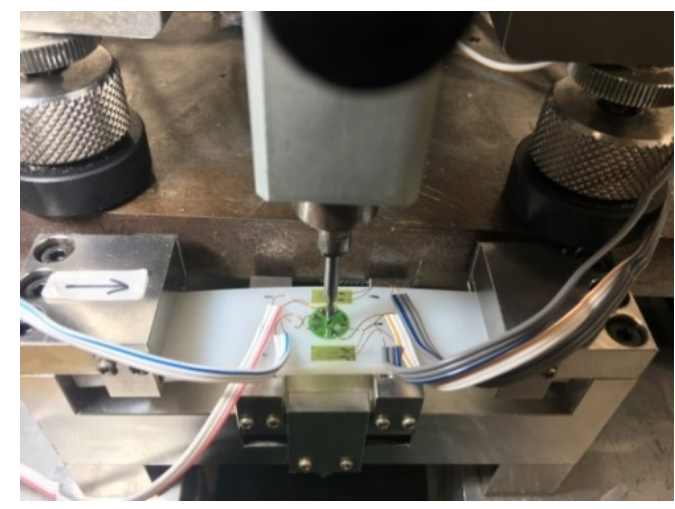

Fig. 1. General view of hole-drilling during four point bending test

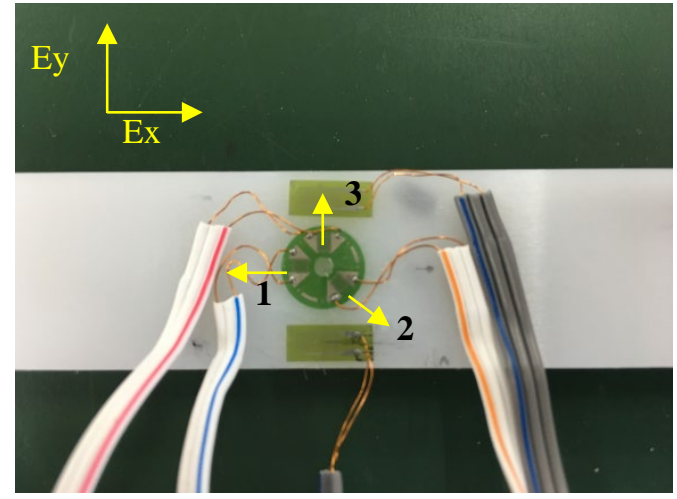

Fig. 2. Rosette strain gage after drilling

Orthotropic elastic properties. The elastic properties of the specimen were measured by using a tensile testing machine. The measured elastic properties are sown in Table. 1.

Where, $E_{x}, E_{y}$ : Elastic moduli along $x$ and $y$ (elastic symmetry) axes

$G_{x y}: x-y$ shear modulus $v_{x y}, v_{y x}: x-y$ Poisson's ratios

Table 1. Elastic properties

\begin{tabular}{|c|c|}
\hline$E_{x}$ & $7.74 \mathrm{GPa}$ \\
\hline$E_{y}$ & $4.83 \mathrm{GPa}$ \\
\hline$G_{x y}$ & $2.26 \mathrm{GPa}$ \\
\hline$v_{x y}$ & 0.365 \\
\hline$v_{y x}$ & 0.287 \\
\hline
\end{tabular}

Hole-drilling measurement. Using the MTS 3000-RESTAN system, a blind hole with a diameter of about $1.8 \mathrm{~mm}$ was drilled at the rosette center of each specimen in each loading condition [see Fig.1 and Fig.2]. The hole was drilled with an increment of $0.05 \mathrm{~mm}$ in the depth direction and the final depth was $1.0 \mathrm{~mm}$ (i.e. $0.05 \mathrm{~mm} \times 20$ steps). At each step, relieved strains were measured and recorded automatically. The measured cumulative relieved strains for the two specimens are shown in Fig.3 and Fig.4. Comparing the relieved strains measured on gage 1(i.e. $x$ direction) in these Figures, they are almost proportional to the given stress ratio of 1.33 (20MPa/15MPa). The final cumulative relieved strains at the depth of $1 \mathrm{~mm}$ were used in the orthotropic analysis described in the next section. On the other hand, all the relieved strains were used in the isotropic analysis in accordance with the ASTM E837 standard. 


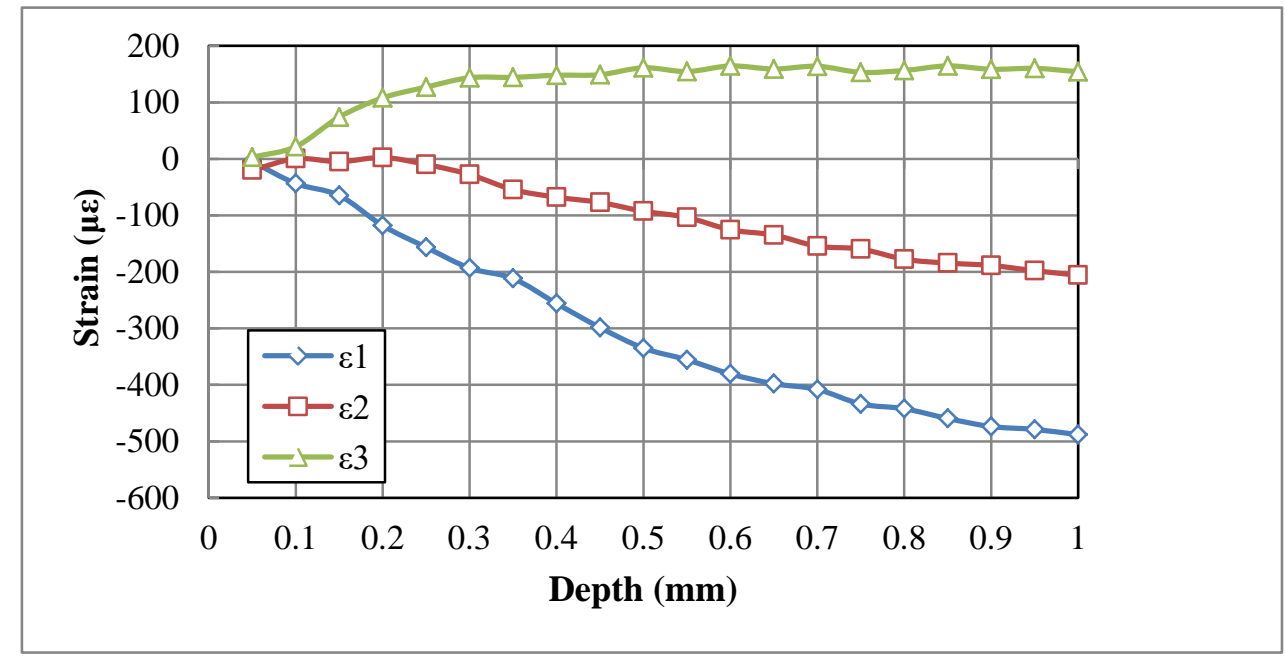

Fig. 3. Measured relieved strains for the applied stress of $15 \mathrm{MPa}$

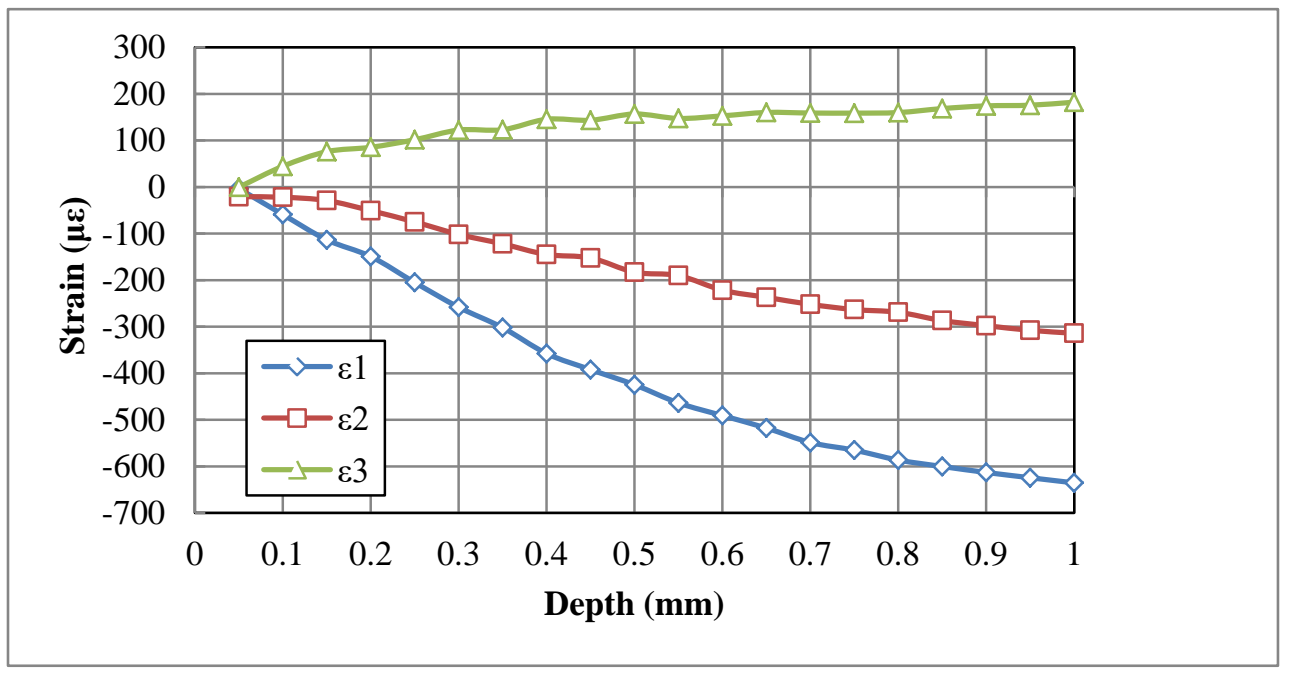

Fig. 4. Measured relieved strains for the applied stress of $20 \mathrm{MPa}$

Orthotropic hole-drilling analysis. We applied the Eq. (1) suggested by Schajer et al [2] for the anisotropic analysis.

$\frac{1}{\sqrt{E_{x} E_{y}}}=\left[\begin{array}{lll}C_{11} & C_{12} & C_{13} \\ C_{21} & C_{22} & C_{23} \\ C_{31} & C_{32} & C_{33}\end{array}\right]\left[\begin{array}{c}\sigma_{x} \\ \tau_{x y} \\ \sigma_{y}\end{array}\right]=\left[\begin{array}{c}\varepsilon_{1} \\ \varepsilon_{2} \\ \varepsilon_{3}\end{array}\right] \quad$ Eq. (1)

Where,

$C_{* *}:$ orthotropic strain relief compliances

$\sigma_{x,} \sigma_{y}: x-y$ Cartesian normal stresses

$\tau_{x y}: x-y$ Cartesian shear stress 
$\varepsilon_{1}, \varepsilon_{2}, \varepsilon_{3}:$ measured relieved strains by rosette strain gage

The values of the elastic compliances in Eq. (1) depend on the orthotropic elastic properties of the specimen, the hole diameter and the rosette strain gage geometry.

Considering the elastic properties in Table 1, the values of the elastic compliances were calculated by using a Table given in [2] and the results are shown in Table 2.

Table 2. The calculated elastic compliances for this study from [2]

\begin{tabular}{|l|l|l|l|l|l|l|}
\hline$C_{11}$ & $C_{13}$ & $C_{21}$ & $C_{22}$ & $C_{23}$ & $C_{31}$ & $C_{33}$ \\
\hline-0.284 & 0.108 & -0.094 & 0.443 & -0.118 & 0.120 & -0.369 \\
\hline
\end{tabular}

Isotropic hole-drilling analysis. We applied an average value of elastic moduli along $x$ and $y$ axes as an isotropic elastic modulus in order to analyze the stress by using the ASTM E837-13 standard.

\section{Results}

The results of the orthotropic and isotropic analyses for uniform stresses with depth are shown in Table 3. In Table 3, 'Applied average stress' means the averaged theoretical bending stress from the surface to the depth of $1 \mathrm{~mm}$ as shown in Fig. 5.

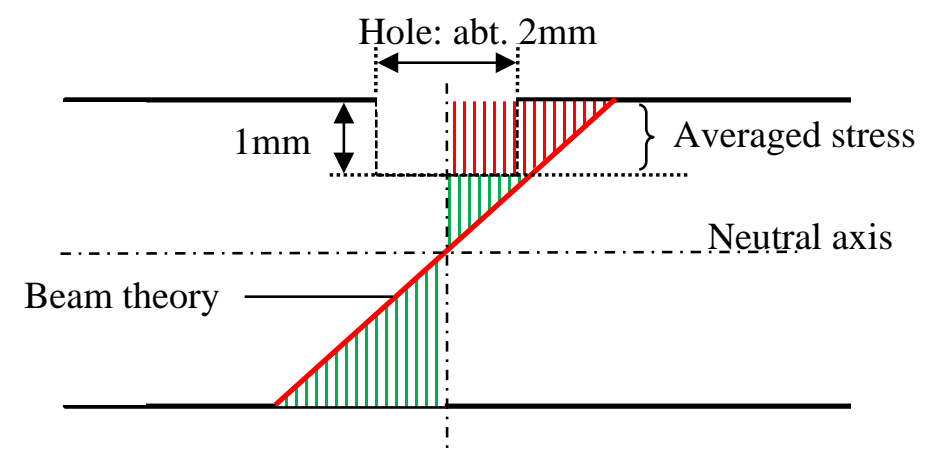

Fig. 5. Stress distribution in the bent specimen and the measured region by hole-drilling method

Table 3. Comparison between applied stresses and calculated stresses by orthotropic and isotropic analyses

\begin{tabular}{|c||c|c|c|}
\hline $\begin{array}{c}\text { Applied bending stress : } \\
\sigma_{\max }(\mathrm{MPa})\end{array}$ & $\begin{array}{c}\text { Applied averaged } \\
\text { stress }: \sigma_{\text {ave }}(\mathrm{MPa})\end{array}$ & $\begin{array}{c}\text { Orthotropic analysis } \\
: \sigma_{x}(\mathrm{MPa})\end{array}$ & $\begin{array}{c}\text { Isotropic analysis }: \sigma_{x} \\
(\mathrm{MPa})\end{array}$ \\
\hline \hline 15.0 & 11.2 & 10.9 & 10.3 \\
\hline 20.0 & 15.0 & 14.4 & 13.7 \\
\hline
\end{tabular}

As shown in Table 3, the uniform stresses calculated by orthotropic and isotropic analyses based on the measured relieved strains agreed well with the applied averaged stresses (i.e. beam theory values).

Furthermore, we calculated a non-uniform stress distribution with depth in accordance with the ASTM E837 standard for the15MPa load case. The result is shown in Fig.6. This result agreed well with the theoretical bending stress distribution based on the beam theory. The deviation 
from the beam theory is considered to be the influence of initial residual stresses present in the specimen. The initial residual stress distribution with depth is considered to be different between specimens. We measured therefore initial residual stress distributions for another three specimens. The measured initial residual stress distributions for $\sigma_{x}$ component are shown in Fig. 7 and it confirms that the initial residual stress distributions are significantly different each other depending on the specimens.

In this study, we applied the plastic specimens with the elastic modulus ratio: $E_{y} / E_{x}$ of 0.62 and confirmed that the effect of the anisotropy on the stress analysis results is not so significant. The elastic modulus ratio: $E_{y} / E_{x}$ of commercially available glass-fiber reinforced plastics is from 0.5 to 0.7 . Consequently, it can be stated that in the residual stress measurement with the holedrilling strain gage method for the glass-fiber reinforced plastics, we can obtain the residual stresses with sufficient accuracy for both "uniform" and "non-uniform" stress cases by applying the averaged elastic properties in the analyses based on ASTM E837 standard.

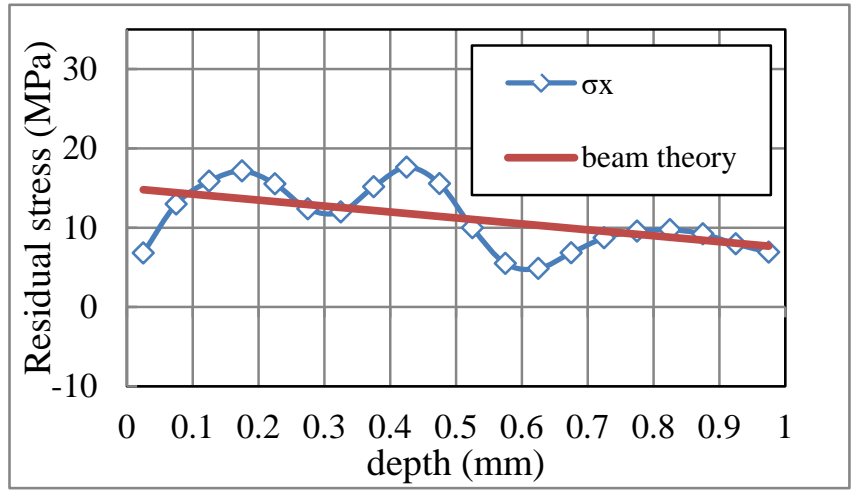

Fig. 6. Comparison between the measured non-uniform stress and the beam theory value for the applied bending stress of $15 \mathrm{MPa}$

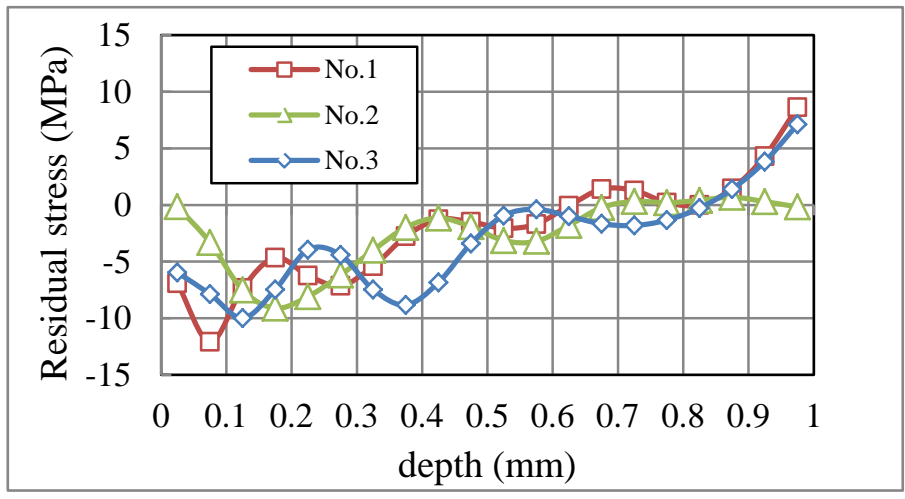

Fig. 7. Measured initial residual stress distributions in another three specimens 


\section{Summary}

Known bending stresses were given by four point bending tests on the rectangular plate specimens made of glass-fiber reinforced plastics with the elastic modulus ratio: $E_{y} / E_{x}$ of 0.62 and the measured stresses by the hole-drilling method were compared with the analyzed values based on the beam theory. As a result, the uniform stresses calculated by orthotropic and isotropic analyses based on the measured relieved strains agreed well with the applied averaged stresses (i.e. beam theory values). In addition, non-uniform stress distribution with depth was calculated in accordance with the ASTM E837 standard and the calculated results agreed well with the theoretical bending stress distribution based on the beam theory for both "uniform” and "non-uniform” stress cases.

\section{References}

[1] ASTM E 837-13a, Standard Test Method for Determining Residual Stresses by the HoleDrilling Strain-Gauge Method 2013.

[2] G.S. Schajer and L. Yang, Residual-stress measurement in orthotropic materials using the hole-drilling method, Exp. Mech., 34, 1994. https://doi.org/10.1007/BF02325147

[3] P. Pagliaro, B. Zuccarello, Residual Stress Analysis of Orthotropic Materials by the Through-hole Drilling Method, Exp. Mech. 47, 2007. https://doi.org/10.1007/s11340-006-9019-3 